\title{
The Number of Blocks of a Graph with Given Minimum Degree
}

\author{
Lei Li $(\mathbb{D})$ and Baoyindureng $\mathrm{Wu}(\mathbb{D}$ \\ College of Mathematics and System Sciences, Xinjiang University Urumqi, Xinjiang 830046, China \\ Correspondence should be addressed to Baoyindureng Wu; baoywu@163.com
}

Received 6 October 2020; Revised 3 January 2021; Accepted 10 January 2021; Published 25 January 2021

Academic Editor: A. E. Matouk

Copyright (c) $2021 \mathrm{Lei} \mathrm{Li}$ and Baoyindureng Wu. This is an open access article distributed under the Creative Commons Attribution License, which permits unrestricted use, distribution, and reproduction in any medium, provided the original work is properly cited.

\begin{abstract}
A block of a graph is a nonseparable maximal subgraph of the graph. We denote by $b(G)$ the number of block of a graph $G$. We show that, for a connected graph $G$ of order $n$ with minimum degree $k \geq 1, b(G)<\left((2 k-3) /\left(k^{2}-k-1\right)\right) n$. The bound is asymptotically tight. In addition, for a connected cubic graph $G$ of order $n \geq 14, b(G) \leq(n / 2)-2$. The bound is tight.
\end{abstract}

\section{Introduction}

We consider finite, undirected, simple graphs only. Let $G=(V(G), E(G))$ be a graph. The numbers of vertices and edges of $G$ are called the order and the size of $G$ and denoted by $v(G)$ and $e(G)$, respectively. A vertex $v$ is called a cut vertex if $\operatorname{com}(G-v)>\operatorname{com}(G)$, where $\operatorname{com}(G)$ denotes the number of components of $G$. $c(G)$ denotes the number of cut vertices of $G$. Rao [1] proved that, for a connected graph $G$ of order $n$ and size $m$,

$$
c(G) \leq \max \left\{q: m \leq\left(\begin{array}{c}
n-q \\
2
\end{array}\right)+q\right\},
$$

characterized all extremal graphs. Rao and Rao [2] solved the corresponding problem for a strong digraph. Later, Achuthan and Rao [3] determined the maximum number of cut edges in a connected $d$-regular graph of order $p$.

Let $f(n, d)=\max \{c(G): G$ is a connected $k$-regular graph of order $n$ \}. Rao [4] determined $f(n, d)$ for $d \leq 4$. Nirmala and Rao [5] showed that $f(n, d)=$ $((2 n-d-5) /(d+1))-1$ or $((2 n-d-5) /(d+1))-2$ for odd $d \geq 5$ and have obtained an upper bound for $f(n, d)$ for even $d \geq 6$.

Alberten and Berman [6] proved that, for a graph $G$ of order $n$ and minimum degree $k \geq 2$,

$$
c(G)<\frac{2 k-2}{k^{2}-2} n \text {. }
$$

This bound is asymptotically tight.

Hopkins and Staton [7] showed that every connected graph of order $n$ contains no more than $(r /(2 r-2)) n$ cut vertices of degree $r$. Some related results are referred to $[8,9]$.

A separation of a connected graph is a decomposition of the graph into two nonempty connected subgraphs which have just one vertex in common. The common vertex is called a separating vertex of the graph. Since the graph $G$ under consideration is simple, $v \in V(G)$ is a separating vertex if and only if it is a cut vertex. A block of a graph is a nonseparable maximal subgraph of the graph. We denote by $b(G)$ the number of blocks of a graph $G$.

It is clear that any two blocks of a graph have at most one vertex in common. Recall that the block tree $B(G)$ of $G$ is the bipartite graph with bipartition $(\mathbb{B}, \mathbb{S})$, where $\mathbb{B}$ is the set of blocks of $G$ and $S$, the set of separating vertices of $G$, and a block $B$, and a separating vertex $v$ is joined by an edge in $B(G)$ if and only if $B$ contains $v$. It is easy to see that if $G$ is connected, $B(G)$ is a tree. Each leaf of $B(G)$ corresponds to an end block of $G$.

Inspired from the bound for the cut vertices, in the present paper, we consider the upper bound for the number of blocks, a connected graph of order $n$ with given minimum degree. Let us begin with two easy cases when $\delta(G)=1$ and $\delta(G)=2$.

Proposition 1. For a connected graph $G$ of order $n \geq 2$, $b(G) \leq n-1$, with equality if and only if $G$ is a tree. 
Proof. Our proof is induction on $n$. If $n=2$, then $G \cong K_{2}$; thus, the result holds. Next, we assume that $n \geq 3$. If $G$ has no cut vertex, then $b(G)=1<n-1$. Now suppose $G$ has a cut vertex. Let $B$ be an end block of $G$ and $v$ be the cut vertex, which belongs to $B$. Let $G^{\prime}=G-(V(B),\{v\})$. Clearly, $G^{\prime}$ is connected. By the induction hypothesis, $b\left(G^{\prime}\right) \leq v\left(G^{\prime}\right)-1$. Since $b(G)=b\left(G^{\prime}\right)+1, \quad v\left(G^{\prime}\right) \leq v(G)-1$, we have $b(G) \leq n-1$, with equality only if $b\left(G^{\prime}\right)=v\left(G^{\prime}\right)-1=n-2$ and $B \cong K_{2}$. By the induction hypothesis, $G^{\prime}$ is a tree, implying that $G$ is a tree.

On the contrary, if $G$ is a tree, clearly, $b(G)=n-1$.

Proposition 2. For a connected graph $G$ of order $n \geq 4$ with $\delta(G) \geq 2, b(G) \leq n-3$, with equality if and only if $G$ is the graph obtained from $P_{n-4}$ identifying each end with a vertex of separate $K_{3}$, as given in Figure 1.

Proof. If $G$ has no cut vertex, the result holds trivially. Next, we assume that $G$ has cut vertices, and thus, it has at least two end blocks and $n \geq 5$. Let $B_{1}, \ldots, B_{t}$ be all end blocks of $G$. Let $c_{i}$ be the cut vertex of $G$, which belongs to $B_{i}$ for each $i \in\{1, \ldots, t\}$. Clearly, $v\left(B_{i}\right) \geq 3$ for any $i$. If $c_{i}=c_{j}$ for any two distinct $i, j$, then $b(G)=t$ and $n=\sum_{i=1}^{t}\left(v\left(B_{i}\right)-\right.$ $1)+1 \geq 2 t+1$. Therefore, $b(G) \leq(n-1) / 2 \leq n-3$.

Otherwise, $G$ has at least two cut vertices. It follows that the order $n^{\prime}$ of $G^{\prime}=G-\cup_{i=1}^{t} V\left(B_{i}\right),\left\{c_{i}\right\}$ is at least two. Hence, $n \geq n^{\prime}+2 t$ and $b(G)=b\left(G^{\prime}\right)+t$. By Proposition 1 , $b\left(G^{\prime}\right) \leq n^{\prime}-1$. Summing up the above, we have

$$
b(G)=b\left(G^{\prime}\right)+t \leq n^{\prime}-1+t \leq n-t-1 \leq n-3 .
$$

From the above, $b(G)=n-3$ if and only if $t=2$ and $G^{\prime}=P_{n-4}, B_{1} \cong K_{3} \cong B_{2}$, as we promised.

It is clear that, for a graph $G$ of order $n, b(G)$ decreases when $\delta(G)$ increases. For a connected graph $G$ of order $n$ and minimum degree at least $k$, we have the following result, which is asymptotically best possible.

Theorem 1. For a connected graph $G$ of order $n$ with $\delta(G) \geq k, b(G)<\left((2 k-3) /\left(k^{2}-k-1\right)\right) n$.

We show that the bound in the above theorem is asymptotically best possible. Let $k \geq 3$. Consider a tree $T$ of order $p$ with each vertex having degree $k$ or 1 . By the handshaking lemma, the number of leaves of this tree is

$$
\frac{(k-2) p+2}{k-1}
$$

Let $G$ be the graph obtained from identifying each leaf of $T$ with a vertex of a clique of order $k+1$ separately. Therefore,

$$
\begin{aligned}
& v(G)=p+\frac{(k-2) p+2}{k-1} k, \\
& b(G)=p-1+\frac{(k-2) p+2}{k-1} .
\end{aligned}
$$

So, we have

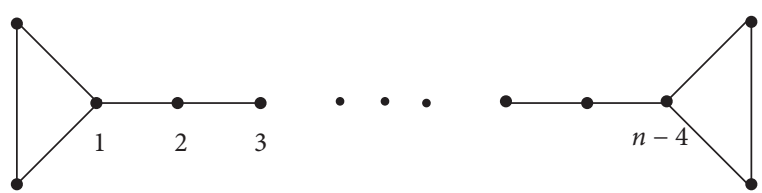

Figure 1: The extremal graph attaining the upper bound in Proposition 2.

$$
\frac{b(G)}{v(G)}=\frac{(2 k-3) p-k+3}{\left(k^{2}-k-1\right) p+2 k}<\frac{2 k-3}{k^{2}-k-1} .
$$

However, as $p$ gets larger, $b(G) / v(G)$ gets arbitrarily close to $(2 k-3) /\left(k^{2}-k-1\right)$.

What happens for the $k$-regular graphs? The situation becomes complicated. We are just able to get an exact bound for a cubic graph $G$ of order $n: b(G)<n / 2$ (Theorem 2), whereas by Theorem 1 , we have $b(G)<(3 / 5) n$ for a connected graph $G$ of order $n$ with $\delta(G) \geq 3$.

Theorem 2. For a connected cubic graph $G$ of order $n$,

$$
b(G) \leq \begin{cases}1, & \text { if } n \leq 8 \\ 3, & \text { if } n=12 \\ \frac{n}{2}-2, & \text { otherwise. }\end{cases}
$$

The bound is sharp.

To see the sharpness of the bound, we denote by $K_{4}^{*}$ the graph obtained from $K_{4}$ by replacing an edge with a path of length two, as drawn in Figure 2.

The graphs $G_{n}$ achieve the upper bound in Theorem 2, which are classified into three types in terms of $n \equiv 4(\bmod$ $6), n \equiv 0(\bmod 6)$, and $n \equiv 2(\bmod 6)$, respectively.

For an integer $n \equiv 4(\bmod 6), k=(n-4) / 3$ is an even integer. Let $T_{k}$ be a tree in which every vertex has degree 1 or 3 . It is clear that $T_{k}$ has exactly $(k+1) / 2$ vertices of degree 1 (leaves) and $(k-1) / 2$ vertices of degree 3 . Let $G_{n}$ be a graph obtained from identifying each leaf of $T_{k}$ with the vertex of degree two of a separate $K_{4}^{*}$, as shown in Figure 3.

For an integer $n \equiv 0(\bmod 6)$, let $G_{n}$ be a cubic graph obtained from a graph $G_{n-2}$ by replacing a vertex of degree three (not belongs to any $K_{4}^{*}$ ) with a triangle, as shown in Figure 3.

For an integer $n \equiv 2(\bmod 6)$, let $G_{n}$ be a cubic graph obtained from a graph $G_{n-4}$ by inserting a $K_{4}-e$ into an edge of $G_{n-4}$ (not belongs to any $K_{4}^{*}$ ), as shown in Figure 3.

It can be checked that $v\left(G_{n}\right)=n$ and $b\left(G_{n}\right)=(n / 2)-2$ for any graph $G_{n}$ constructed as above.

\section{The Proof of Theorem 1}

Suppose the result is not true and let $G$ be a counterexample of minimum order $n$, i.e., $\delta(G) \geq k$ and $b(G) \geq((2 k-3)$ / $\left.\left(k^{2}-k-1\right)\right) n$, but for any connected graph $G^{\prime}$ of order $n^{\prime}<n$ with $\delta\left(G^{\prime}\right) \geq k, b\left(G^{\prime}\right)<\left((2 k-3) /\left(k^{2}-k-1\right)\right) n^{\prime}$. 


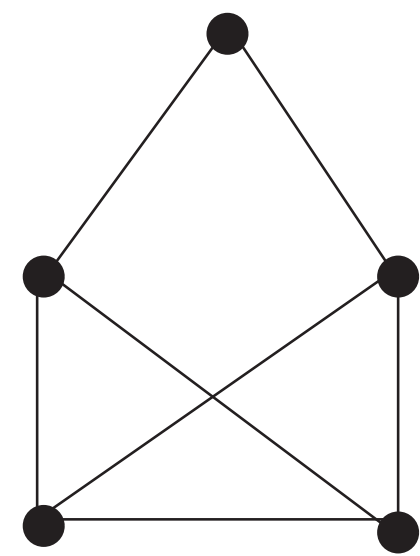

Figure $2: K_{4}^{*}$.

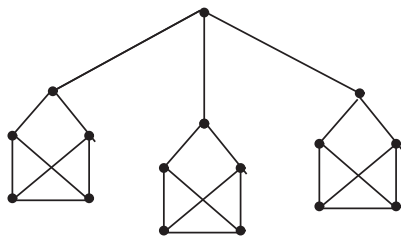

(a)

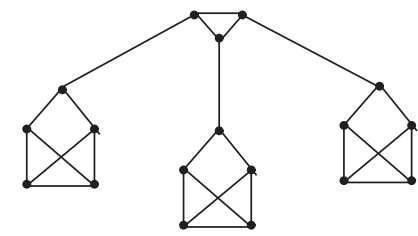

(b)

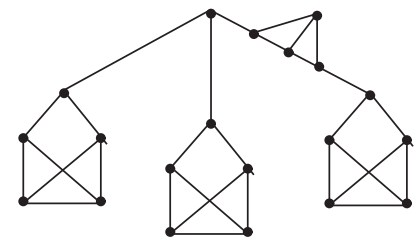

(c)

Figure 3: The extremal graph $G$ of order $n$ attaining the upper bound in Theorem 2, where (a) $n \equiv 4$, (b) $n \equiv 0$, and (c) $n \equiv 2$ (mod 6), respectively.

If $k \in\{1,2\},\left((2 k-3) /\left(k^{2}-k-1\right)\right) n=n$. By Propositions 1 and $2, b(G) \leq n-1<n$. Hence, $k \geq 3$. Since $n \geq k+1$, we have $\left((2 k-3) /\left(k^{2}-k-1\right)\right) n \geq 1$, and thus, $G$ has at least two blocks.

Claim 1. Every end block of $G$ is a clique of order $k+1$.

Proof of Claim 1. If it is not, let $B$ be an end block of $G$. Let $G^{\prime}$ be the graph obtained from $G$ by replacing $B$ with $B^{\prime}$ of order $k+1$. Clearly, $b(G)=b\left(G^{\prime}\right)$ and $\delta\left(G^{\prime}\right) \geq k$. By the choice of $G$,

$$
b\left(G^{\prime}\right)<\frac{2 k-3}{k^{2}-k-1} n^{\prime} .
$$

Combining the above facts, we conclude that $b(G)<\left((2 k-3) /\left(k^{2}-k-1\right)\right) n$, contradicting the choice of G.

Claim 2. No cut vertex of $G$ belongs to at least two end blocks of $G$.

Proof of Claim 2. Let $B$ and $B^{\prime}$ be two end blocks of $G$ containing the same cut vertex $v$ of $G$. Let $G^{\prime}=G-\left(V\left(B^{\prime}\right),\{v\}\right)$. By Claim $1, v(B)=v\left(B^{\prime}\right)=k+1$, and thus, $v\left(G^{\prime}\right)=n-k$ and $\delta\left(G^{\prime}\right) \geq k$. By the minimality of G,

$$
b\left(G^{\prime}\right)<\frac{2 k-3}{k^{2}-k-1}(n-k) .
$$

Combining (9) with the fact that $b(G)=b\left(G^{\prime}\right)+1$, we have a contradiction:

$$
b(G)<\frac{2 k-3}{k^{2}-k-1}(n-k)+1<\frac{2 k-3}{k^{2}-k-1} n,
$$

Claim 3. Let $c$ be a cut vertex lying on an end block $B_{c}$. If $B \neq B_{c}$ is a block containing $c$, then $B \cong K_{2}$.

Proof of Claim 3. It suffices to show that $v(B)=2$.

First suppose that $v(B) \geq k+1$. Let $G^{\prime}$ be the graph obtained from $G,\left(V\left(B_{c}\right),\{c\}\right)$ and joining $c$ to every vertex in $V(B),\{c\}$. Clearly, $G^{\prime}$ is a connected graph with $\delta\left(G^{\prime}\right) \geq k$. Moreover, by Claim 1, $v\left(G^{\prime}\right)=n-k$. Again, by the minimality of $G$,

$$
b\left(G^{\prime}\right)<\frac{2 k-3}{k^{2}-k-1}(n-k) .
$$

Combining (11) with the fact that $b(G)=b\left(G^{\prime}\right)+1$, we have a contradiction.

$$
b(G)<\frac{2 k-3}{k^{2}-k-1}(n-k)+1<\frac{2 k-3}{k^{2}-k-1} n,
$$

Now assume that $v(B) \in\{3, \ldots, k\}$. Let $V(B)=\left\{v_{1}, \ldots, v_{r}\right\}$, where $v_{1}=c$. Since $\delta(G) \geq k$ and $r \leq k$, each $v_{i}$ is a cut vertex of $G$. Let $G^{\prime}$ be the graph obtained from $G$ by identifying all vertices in $\left\{v_{2}, \ldots, v_{r}\right\}$. Clearly, $\delta\left(G^{\prime}\right) \geq k, n^{\prime}<n$, and $b\left(G^{\prime}\right)=b(G)$. By the choice of $G$, $b\left(G^{\prime}\right)<\left((2 k-3) /\left(k^{2}-k-1\right)\right) n^{\prime}$. Thus, 


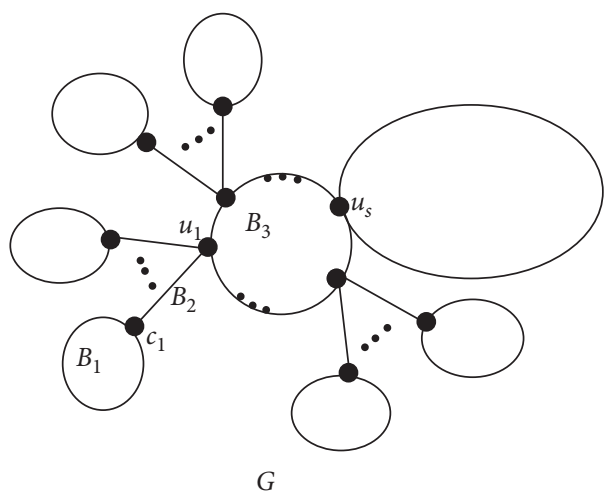

(a)

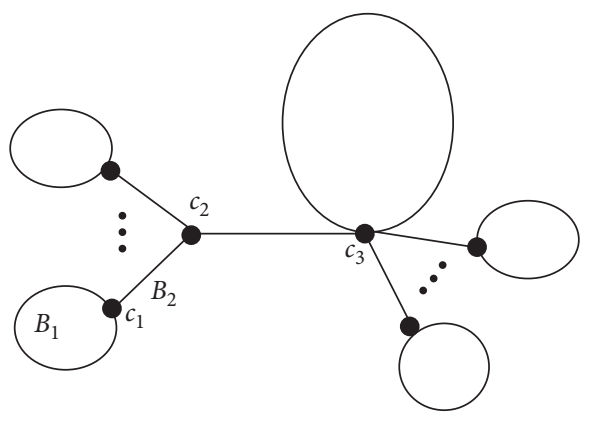

$G^{\prime}$

(b)

Figure 4: (a) $G$ and (b) $G^{\prime}$ in case 3.2.

$$
b(G)<\frac{2 k-3}{k^{2}-k-1} n,
$$

contradicting the choice of $G$. This proves the claim.

Take a longest path $P$ of $B(G)$. Let $B_{1}$ be an end block of $G$, which corresponds to a terminal vertex of $P=B_{1} c_{1} B_{2} c_{2} B_{3} c_{3} \cdots$, where $c_{1}$ be the unique cut vertex of $G$ which belongs to $B_{1}$. By Claim $3, B_{2} \cong K_{2}$. Next, we consider three possible cases in terms of the order $v\left(B_{3}\right)$ of $B_{3}$.

2.1. Case 1: $v\left(B_{3}\right) \geq k+1$. Let $G^{\prime}$ be the graph obtained from $G, V\left(B_{1}\right)$ and joining $c_{2}$ to each vertex of $V\left(B_{3}\right)$. It is clear that $\delta\left(G^{\prime}\right) \geq k$, and by Claim $1, v\left(G^{\prime}\right)=n-k-1$. By the minimality of $G, b\left(G^{\prime}\right)<\left((2 k-3) /\left(k^{2}-k-1\right)\right)(n-k-1)$. Since $b(G)=b\left(G^{\prime}\right)+2$, we have

$$
b(G)<\frac{2 k-3}{k^{2}-k-1}(n-k-1)+2<\frac{2 k-3}{k^{2}-k-1} n .
$$

2.2. Case 2: $v\left(B_{3}\right)=2$. By the choice of $P$, each block $B^{*} \neq B_{3}$ of $G$ containing $c_{2}$ is isomorphic to $K_{2}$. In addition, the end block containing the other end of $B^{*}$ is a leaf of $B(G)$. Since $d\left(c_{2}\right) \geq k$, there are $k-1$ such end block $B_{1}^{*}, \ldots, B_{k-1}^{*}$, each of which are jointed $c_{2}$ with an edge. Let $G^{\prime}$ be the graph obtained from $G-\cup_{i=1}^{k-1} V\left(B_{i}^{*}\right)$ by identifying a vertex of a new clique of order $k+1$ with $c_{2}$. It is clear that $\delta\left(G^{\prime}\right) \geq k$, $n^{\prime}=v\left(G^{\prime}\right)=n-(k-1)(k+1)+k$, and $b\left(G^{\prime}\right)=b(G)-2$ $(k-1)+1$. So,

$$
\begin{aligned}
b(G) & =b\left(G^{\prime}\right)+2(k-1)-1 \\
& <\frac{2 k-3}{k^{2}-k-1} n^{\prime}+2(k-1)-1 \\
& =\frac{2 k-3}{k^{2}-k-1}(n-(k-1)(k+1)+k)+2(k-1)-1 \\
& =\frac{2 k-3}{k^{2}-k-1} n
\end{aligned}
$$

is a contradiction.
2.3. Case 3: $3 \leq v\left(B_{3}\right) \leq k$. Since $\delta(G) \geq k$, each vertex of $B_{3}$ is a cut vertex of $G$. We distinguish two subcases in terms of $v\left(B_{3}\right)$.

2.3.1. Case 3.1: $v\left(B_{3}\right)=k$. Since $\delta(G) \geq k$, every vertex $v \in V\left(B_{3}\right)$ has a neighbor not in $V\left(B_{3}\right)$, which belongs to distinct blocks of $G$. Let $G^{\prime}$ be the graph obtained from $G$ by contracting $B_{3}$ to a vertex $v^{\prime}$. It can be seen that $\delta\left(G^{\prime}\right) \geq k$, $b(G)=b\left(G^{\prime}\right)+1$, and $n^{\prime}=n-k+1$. So,

$$
b(G)=b\left(G^{\prime}\right)+1<\frac{2 k-3}{k^{2}-k-1}(n-k+1)+1<\frac{2 k-3}{k^{2}-k-1} n .
$$

2.3.2. Case 3.2: $3 \leq v\left(B_{3}\right) \leq k-1$. Let $s=v\left(B_{3}\right)$ and $V\left(B_{3}\right)=\left\{u_{1}, \ldots, u_{s}\right\}$, where $u_{1}=c_{2}$ and $u_{s}=c_{3}$. Since $\delta(G) \geq k$, for any $i \in\{1, \ldots, s-1\}$, there are at least $k-s+1$ blocks containing $u_{i}$, each of which is isomorphic to $K_{2}$, as illustrated in Figure 4.

Let $G^{\prime}$ be the graph obtained from joining each component of $G-\left\{u_{2}, \ldots, u_{s-1}\right\}$ to $c_{2}$ or $c_{3}$ such that $d_{G^{\prime}}\left(c_{j}\right) \geq k$ for each $j \in\{2,3\}$. In addition, add an edge $c_{2} c_{3}$ if $c_{2} c_{3} \notin E(G)$. Note that $G^{\prime}$ is a connected graph of order $n^{\prime}<n$ with $\delta\left(G^{\prime}\right) \geq k$ and $b\left(G^{\prime}\right)=b(G)$. By the minimality of $G, \quad b\left(G^{\prime}\right)<\left((2 k-3) /\left(k^{2}-k-1\right)\right) n^{\prime}$. Therefore, $b(G)<$ $\left((2 k-3) /\left(k^{2}-k-1\right)\right) n$, contradicting the choice of $G$.

The proof of Theorem 1 is completed.

\section{Proof of Theorem 2}

Suppose the result is not true and let $G$ be a counterexample of minimum order $n$. The following fact is clear:

(1) $G$ must contain cut vertex.

Since no cubic graph of order $\leq 8$ has a cut vertex, $n \geq 10$.

(2) Moreover, $n \geq 14$. If $10 \leq n \leq 12$, it is not hard to check that $b(G) \leq 3$.

Claim 4. Every end block of $G$ is a $K_{4}^{*}$. 
Proof of Claim 4. If it is not, let $B$ be an end block of $G$. Let $G^{\prime}$ be the graph obtained from $G$ by replacing $B$ with $K_{4}^{*}$. Clearly, $G^{\prime}$ is a connected cubic graph of order $n^{\prime}<n$ and $b\left(G^{\prime}\right)=b(G)$. By the minimality of $G$,

$$
b\left(G^{\prime}\right) \leq \frac{n^{\prime}}{2}-2 \text {. }
$$

Combining the above facts, we conclude that $b(G) \leq(n / 2)-2$, contradicting the choice of $G$.

Take a longest path $P$ of $B(G)$. Let $B_{1}$ be an end block of $G$, which corresponds to a terminal vertex of $P=B_{1} c_{1} B_{2} c_{2} B_{3} c_{3} \cdots$, where $c_{1}$ be the unique cut vertex of $G$ which belongs to $B_{1}$. Since $G$ is a cubic graph, $B_{2} \cong K_{2}$. Next we consider three possible cases.

3.1. Case 1: $v\left(B_{3}\right)=k \geq 4$. If $v \in V\left(B_{3}\right)$ is a cut vertex of $G$, then $v$ belongs to another block which is isomorphic to $K_{2}$. In addition, the end block containing the other end of the $K_{2}$ is a leaf of $B(G)$. We may assume $B_{3}$ has $r$ cut vertices except $c_{3}$, which belong to $B_{1}^{\prime}, B_{2}^{\prime}, \ldots, B_{r}^{\prime}$, respectively, where $B_{1}^{\prime}=B_{1}$.

Let $G^{\prime}$ be the graph obtained from $G-\cup_{i=1}^{r} V\left(B_{i}^{\prime}\right)-$ $\left(V\left(B_{3}\right),\left\{c_{3}\right\}\right)$ by identifying $c_{3}$ with the vertex of degree two of a new $K_{4}^{*}$. It is clear that $G^{\prime}$ is a cubic graph of order $n^{\prime}=n-5 r-k+5$ and $b\left(G^{\prime}\right)=b(G)-2 r$. By the induction hypothesis, $b\left(G^{\prime}\right) \leq(1 / 2)(n-5 r-k+5)-2$. Thus,

$$
\begin{aligned}
b(G) & =b\left(G^{\prime}\right)+2 r \\
& \leq \frac{1}{2}(n-5 r-k+5)-2+2 r \\
& =\frac{n}{2}-\frac{r}{2}-\frac{k}{2}+\frac{1}{2}, \quad(k \geq 4), \\
& \leq \frac{n}{2}-2+\frac{1}{2}-\frac{r}{2} \\
& \leq \frac{n}{2}-2 .
\end{aligned}
$$

3.2. Case 2: $v\left(B_{3}\right)=3$. It follows that $B_{3} \cong K_{3}$. Every vertex of $B_{3}$ is cut vertex. Let $G^{\prime}$ be the graph obtained by the same operation as in the proof of Case 1 . We have $n^{\prime}=n-8$ and $b\left(G^{\prime}\right)=b(G)-4$. Therefore,

$$
b(G)=b\left(G^{\prime}\right)+4 \leq \frac{1}{2}(n-8)-2+4=\frac{1}{2} n-2 .
$$

3.3. Case 3: $v\left(B_{3}\right)=2$. By the choice of $P$ and $v\left(B_{3}\right)=2$, one can find another longest path $P^{\prime}=B_{1}^{\prime} c_{1}^{\prime} B_{2}^{\prime} c_{2} B_{3} c_{3} \cdots$ of $B(G)$. Let $G^{\prime}$ be the graph obtained from identifying $c_{2}$ of $G-V\left(B_{1}\right)-V\left(B_{1}^{\prime}\right)$ with the vertex of degree two of a new $K_{4}^{*}$. Note that $b\left(G^{\prime}\right)=b(G)-3$ and $n^{\prime}=n-6$. By the induction hypothesis, $b\left(G^{\prime}\right) \leq\left(n^{\prime} / 2\right)-2$. Therefore,

$$
b(G)=b\left(G^{\prime}\right)+3 \leq \frac{1}{2}(n-6)-2+3=\frac{1}{2} n-2 .
$$

The proof is completed.

\section{Conclusions and Future Work}

By arguing the properties of a minimum counterexample to the assertion of the main theorems and by using several kinds of graph transformation, we arrive at a contradiction, and thereby, we show our results. However, the upper bound for $b(G)$ remains open if $G$ is a $k$-regular graph with $k \geq 4$. One of the referees pointed out the possibility of the obtained results to some real-life applications and other fields (see [10-12] for instance).

\section{Data Availability}

No data were used to support this study.

\section{Conflicts of Interest}

The authors declare that they have no conflicts of interest.

\section{Acknowledgments}

The research is supported by the Key Laboratory Project of Xinjiang (2018D04017), NSFC (No. 12061073 and XJEDU2019I001).

\section{References}

[1] A. R. Rao, "An extremal problem in graph theory," Israel Journal of Mathematics, vol. 6, pp. 261-266, 1968.

[2] S. B. Rao and A. R. Rao, "The number of cut verties and cut arcs in a strong directed graph," Acta Mathematica Hungarica, vol. 22, pp. 411-421, 1971.

[3] N. Achuthan and A. R. Rao, "On the number of cut edges in a regular graph," The Australasian Journal of Combinatorics, vol. 27, pp. 5-12, 2003.

[4] A. R. Rao, "Some extremal problems and characterization in the theory of graphs," Ph.D. thesis, Indian Statistical Institute, Chennai, India, , 1969.

[5] K. Nirmala and A. R. Rao, "The number of cutvertices in a regular graph," Centre d'etudes de recherche operationnelle, vol. 17, pp. 295-299, 1975.

[6] M. O. Albertson and D. M. Berman, "The number of cutvertices in a graph of given minimum degree," Discrete Mathematics, vol. 89, no. 1, pp. 97-100, 1991.

[7] G. Hopkins and W. Staton, "On the number of cut-vertices in a graph," International Journal of Mathematics and Mathematical Sciences, vol. 12, no. 2, pp. 305-308, 1989.

[8] G. Chartrand, F. Saba, J. K. Cooper, F. Harary, and C. E. Wall, "Smallest cubic and quartic graphs with a given number of cutpoints and bridges," International Journal of Mathematics and Mathematical Sciences, vol. 5, no. 1, pp. 41-48, 1982.

[9] D. Wang and E. Shan, "On the numbers of cut-vertices and end-blocks in 4-regular graphs," Discuss. Math. Graph Theory, vol. 34, pp. 127-136, 2014.

[10] A. El Atik, A. Nawar, and M. Ate, "Rough approximation models via graphs based on neighborhood systems," Granular Computing, 2020.

[11] A. M. Kozae, A. A. El Atik, A. Elrokh, and M. Atef, "New types of graphs induced by topological spaces," Journal of Intelligent \& Fuzzy Systems, vol. 36, no. 6, pp. 5125-5134, 2019.

[12] S. Nada, A. E. F. El Atik, and M. Atef, "New types of topological structures via graphs," Mathematical Methods in the Applied Sciences, vol. 41, no. 15, pp. 5801-5810, 2018. 